\title{
Nonlinear Optical Measurements of the Artery Wall: Parameters Related to the Progression of Atherosclerosis
}

\author{
${ }^{1}$ Michael G. Sowa, ${ }^{1}$ Leila B. Mostaco-Guidolin, ${ }^{1}$ Michael S.D. Smith, ${ }^{1}$ Elica K. Kohlenberg, \\ ${ }^{2}$ Andrew Ridsdale, ${ }^{2}$ Albert Stolow and ${ }^{1}$ Alex C.T. Ko \\ ${ }^{1}$ Institute for Biodiagnostics, National Research Council Canada, Winnipeg, Canada, R3B 1Y6 \\ ${ }^{2}$ Steacie Institute for Molecular Sciences, National Research Council Canada, Ottawa, Canada, K1A 0R6 \\ email: mike.sowa@nrc-cnrc.gc.ca
}

\begin{abstract}
Nonlinear optical (NLO) microscopy is used to follow key structural and biochemical changes associated with the progression of atherosclerosis. Arteries from WHHL-MI rabbits are examined using a 3 channel NLO microscope that can simultaneously monitor the coherent anti-stokes Raman scattered light (CARS), the two-photon excited fluorescence (TPEF) and the second harmonic generation (SHG) from a sample. Distinct differences in the nonlinear optical signals are observed that correlate with the age of the vessel and the presence of atherosclerotic plaque. These differences are attributed to the changing extracellular matrix and the increased lipid deposition associated with plaque development. The capability of NLO to perform 3D sectioning in thick highly scattering vessels in order to visualize structural details of the artery wall and highlight vessel pathology is demonstrated. These features make NLO a potentially valuable tool to help understand the progression of atherosclerosis.
\end{abstract}

Keywords: Nonlinear optical microscopy, coherent anti-Stokes Raman scattering, two photon excited fluorescence, second harmonic generation, atherosclerosis

\section{INTRODUCTION}

A THEROSCLEROSIS once thought of as a passive disease characterized by the progressive accumulation of fatty deposits in arteries is now recognized as a complex disease where plaque development is mediated by a range of factors.[1] Early consequences of the disease include a thickening of the arterial wall and the appearance of fatty streaks on the vessel. In more advanced disease, vascular smooth muscle cells and a fiborous collagen-elastin network form to encapsulate lipid pools. Superimposed on these pathological changes is the normal aging process that affects vessels. For example, vessels become progressively stiffer with age as a result of changes to the elastin - collagen network within the vessel wall. NLO where elastin, collagen and lipid structures can be simultaneously imaged through the monitoring of two-photon excited fluorescence (TPEF), second harmonic generation ( $\mathrm{SHG}$ ) and coherent antistrokes Raman scattering (CARS) is ideally suited to following these changes in the wall of the artery.[2,3] The technique requires no stains to be added to the tissue and the imaging can be perform in an epi-configuration, obviating the need to prepare the tissue in thin sections. These additional features makes NLO microscopy are very convenient tool to use to study atherogenesis.

\section{SUBJECT AND METHODS}

\section{Nonlinear Optical Microscopy}

As discussed in detail in Pegoraro et al, [4] a multiphoton laser scanning microscope system based on the schematic in Fig.1 was built to study bulk arterial tissue samples. A Ti:Sapphire oscillator (Spectra-Physics, Tsunami) centered at $800 \mathrm{~nm}$ with a pulse width of $100 \mathrm{fs}$, average output power of $1 \mathrm{~W}$ when pumped with $7.25 \mathrm{~W}$ green laser at $532 \mathrm{~nm}$

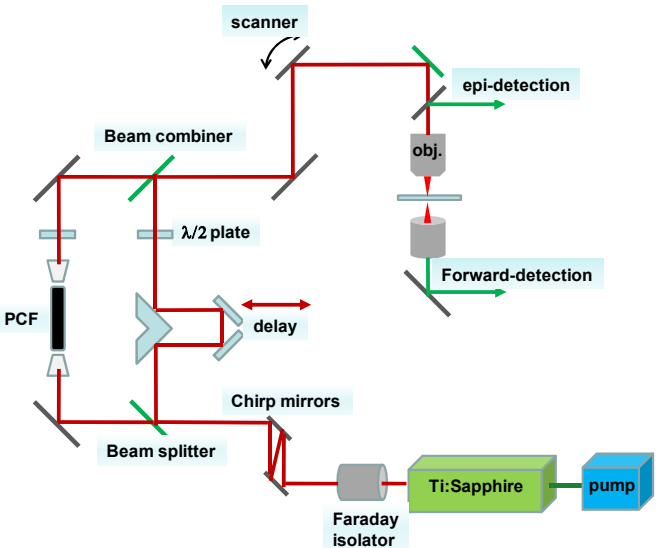

Fig.1 Schematic of the nonlinear optical microscope with the Stokes pulses being generated in PCF which is pumped by the same Ti:sapphire laser that provides the pump pulse.

(Spectra-Physics, Millennium Pro) was used as the laser source. The output femto-second laser pulses were first passed through a Faraday isolator (Newport), re-compressed using a pair of GTI laser mirrors (Layertec $\mathrm{GmbH}$, Germany) and then split into reflected pulses (pump) and the transmitted pulses (Stokes), using a 50/50 beam splitter. An objective lens focused the transmitted pulses into a photonic crystal fiber (FemtoWhite 800, Crystal Fibre, Denmark) to generate broadband emission where the NIR portion of the broadband emission was retained as the Stokes pulses for generating the CARS signal. The pump and Stokes pulses were combined and sent into the microscope assembly where three nondescanned modular type PMT detectors (Hamamatsu) were used for the simultaneous detection of TPEF, SHG and CARS signals in the epi-direction. The laser pulses were focused 
onto sample through a 20x, 0.75 NA infinity corrected air objective lens (Olympus) with the emitted TPEF and epiSHG/CARS signals being collected through the same objective lens. ScanImage (Cold Spring Harbour, NY) was used for laser scanning control and image acquisition and Image J ver $1.42 \mathrm{~b}$ was used for post image processing and image viewing.

\section{Arterial tissue samples and sample preparation}

All animal experiments conformed to the guidelines set out by the Canadian Council on Animal Care regarding the care and use of experimental animals and were approved by the local Animal Care Committee of the National Research Council of Canada. The myocardial infarction prone Watanabe heritable hyperlipidemic (WHHL-MI) rabbits spontaneously develop atherosclerotic plaques due to a hereditary defect in LDL processing.[5,6] This model was used to study plaque development where the aorta was dissected from the ascending aorta to the external iliac artery and then rinsed in heparinized saline. The exterior aorta was subdivided into $\sim 20-30 \mathrm{~mm}$ sections resulting in 5-7 pieces that were cut open longitudinally exposing the luminal surface. The samples were placed in petri dishes with the luminal surface facing up on a moist surface and hydration was maintained throughout the measurements by applying PBS solution periodically. Digital photos of the luminal surface were acquired and regions of interest were identified prior to nonlinear optical measurements.

\section{RESUlTS \& DisCUSSION}

Representative NLO measurements from the luminal surface of a healthy aorta are presented in Figure 2 and compared to images in Fig. 3 taken from a region of older aorta with atherosclerotic plaque.

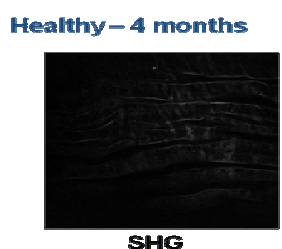

SHG

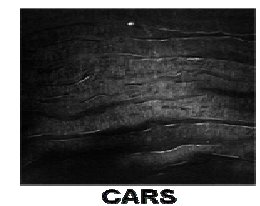

CARS

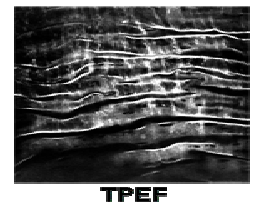

TPEF

Fig.2 Second harmonic generation (SHG), coherent anti-stokes Raman scattered (CARS) and two photon excited fluorescence (TPEF) images of healthy vessel.

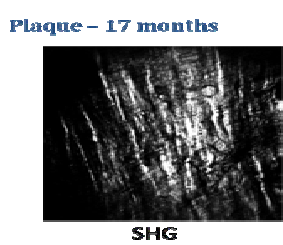

SHG

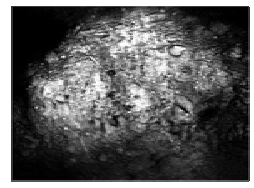

CARS

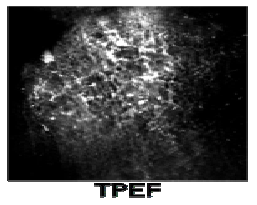

Fig.3 Second harmonic generation (SHG), coherent anti-stokes Raman scattered (CARS) and two photon excited fluorescence (TPEF) images of older vessel with plaque.

Distinct differences can be observed in the TPEF, SHG and CARS images of young and old vessels. The TPEF images of the surface of young, healthy vessels show an elastin membrane structure with relatively weak SHG and CARS signal indicating a limited presence of type I collagen and lipids at the surface of the vessel. With age, the texture of the TPEF image changes revealing a dense interconnected elastin network but also with much higher SHG indicating a greater contribution from collagen. In addition, from regions with plaque an intense CARS lipid signal is observed. Simple parameterizations of these images in terms of texture and intensity can be used to quantify these differences.

\section{CONCLUSIONS}

Nonlinear optical imaging of the two-photon excited fluorescence and second harmonic generation in arterial tissue is demonstrated to be a useful tool for understanding the elastin-collagen network of vessels. The organization of the network is shown to change with age and with the advancement of atherosclerotic disease. In addition, coherent antistrokes Raman scattering can be used to follow lipid deposition in the wall of the artery.

\section{ACKNOWLEDGEMENTS}

The authors acknowledge Vijay Iyer of Cold Spring Harbour Laboratory (NY,USA) for assistance with the ScanImage software. This work was supported by National Research Council Canada, Genomics and Health Initiative.

\section{REFERENCES}

[1] Libby, P. (2006). Atherosclerosis: disease biology affecting the coronary vasculature. Am. J. Cardiol., 98 (12A), 3Q-9Q.

[2] Le, T.T., Langohr, I.M., Locker, M.J., Sturek, M., Cheng, J.-X. (2007). Label-free molecular imaging of atherosclerotic lesions using multimodal nonlinear optical microscopy. J. Biomed. Opt., 12 (5), 054007.

[3] Ko, A.C.T., Ridsdale, A., Pegoraro, A.F., Smith, M.S.D., Mostaço-Guidolin, L.B., Hewko, M.D., Kohlenberg, E.M., Schattka, B.J., Shiomi, M., Stolow, A., Sowa, M.G. (2009). Label-free imaging of arterial tissues using photonic crystal fiber (PCF) based nonlinear optical microscopic system. In Imaging, Manipulation, and Analysis of Biomolecules, Cells, and Tissues VII. Proc. SPIE. Vol. 7182. Bellingham: SPIE, 718204.

[4] Pegoraro, A.F., Ridsdale, A., Moffatt, D.J., Jia, Y., Pezacki, J.P., Stolow, A. (2009). Optimally chirped multimodal CARS microscopy based on a single Ti:sapphire oscillator. Optics Express, 17 (4), 2984-96.

[5] Shiomi, M., Ito, T., Yamada, S., Kawashima, S., Fan, J. (2004). Correlation of vulnerable coronary plaques to sudden cardiac events. Lessions from a myocardial infarction - prone animal model (the WHHLMI rabbit). J. Atheroscler. Thromb., 11 (4), 184-9.

[6] Shiomi, M., Ito, T., Yamada, S., Kawashima, S., Fan, J. (2003). Development of an animal model for spontaneous myocardial infarction (WHHLMI rabbit). Arterioscler. Thromb. Vasc. Biol., 23 (7), 1239-44. 